\title{
Study on Combined shell Mechanics Analysis
}

\author{
Xiangzhong Meng \\ College of Marine, Northwestern Polytechnical University, Xi' an 710072, China \\ Tel: 86-29-8847-4122 E-mail: mengxz@mail.nwpu.edu.cn \\ Xiuhua Shi \& Xiangdang Du \\ College of Marine, Northwestern Polytechnical University, Xi'an, 710072, China
}

The research is supported by graduate starting seed foundation of Northwestern Polytechnical University. No. Z200510.

\begin{abstract}
The AUV combines mostly in ball shell, cylindrical shell, taper shells and other rotary shells by thread coupling, bolt coupling, wedge coupling and hoop coupling. This paper makes the finite element analysis and research on the mechanics mode of a certain AUV with the analytic method. Based on the basic equation of theory of thin shells, analysed every separated shells, and set up it's mechanics mathematical model, and analysed the combined shell with the finite element method. At last, the final result validated the mathematical model. The method presented is effective in analysing and dynamical designing of AUV structure.
\end{abstract}

Keywords: Combined shell, Mathematical model, FEA

During the work progress of AUV, such as torpedo and mine, the shell endures the hydraulic pressure. The research of vibration has important theory value and practical meaning on AUV. The AUV is combines mostly in ball shells, column shells, taper shells and other rotary shells by thread coupling, bolt coupling, wedge coupling and hoop coupling. It is shown in figure 1.

\section{The basic theoretical equation of thin shell}

A middle surface patch of thin shell and internal forces on the cross section are shown in figure 2. The parameters $N_{1}, N_{2}, N_{12}, M_{1}, M_{2}, M_{12}, Q_{1}, Q_{2}$ are the internal forces acted on $\alpha$ plane and $\beta$ plane, $k_{1}$ and $k_{2}$ are the main curvatures on $\alpha$ direction and $\beta$ direction, $R_{1}$ and $R_{2}$ are the radius of main curvature on the middle surface, and $k_{1}=1 / R_{1}, k_{2}=1 / R_{2}$, $\mathrm{A}$ and $\mathrm{B}$ are the Lame coefficients on $\alpha$ direction and $\beta$ direction, $p_{1}, p_{2}, p_{3}$ are the component of loads on $\alpha$ direction, $\beta$ direction and $\gamma$ direction, $u, v$ and $w$ are the component of displacements on $\alpha$ direction, $\beta$ direction and $\gamma$ direction of any point on the middle surface of shell.

The balanceable equations of basic equation in the thin shell theory are:

$$
\left.\begin{array}{l}
\frac{\partial}{\partial \alpha}\left(B N_{1}\right)-\frac{\partial B}{\partial \alpha} N_{2}+\frac{\partial A}{\partial \beta} N_{12}+\frac{\partial}{\partial \beta}\left(A N_{12}\right)+A B k_{1} Q_{1}+A B p_{1}=0 \\
\frac{\partial}{\partial \beta}\left(A N_{2}\right)-\frac{\partial A}{\partial \beta} N_{1}+\frac{\partial B}{\partial \alpha} N_{12}+\frac{\partial}{\partial \alpha}\left(B N_{12}\right)+A B k_{2} Q_{2}+A B p_{2}=0 \\
\frac{\partial}{\partial \alpha}\left(B M_{12}\right)+\frac{\partial B}{\partial \alpha} M_{12}-\frac{\partial A}{\partial \beta} M_{1}+\frac{\partial}{\partial \beta}\left(A M_{2}\right)-A B Q_{2}=0 \\
\frac{\partial}{\partial \beta}\left(A M_{12}\right)+\frac{\partial A}{\partial \beta} M_{12}-\frac{\partial B}{\partial \alpha} M_{2}+\frac{\partial}{\partial \alpha}\left(B M_{1}\right)-A B Q_{1}=0 \\
-A B\left(k_{1} N_{1}+k_{2} N_{2}\right)+\frac{\partial}{\partial \alpha}\left(B Q_{1}\right)+\frac{\partial}{\partial \beta}\left(A Q_{2}\right)+A B p_{3}=0
\end{array}\right\}
$$

From the geometrical equations (1.2) and the physical equations (1.3) of basic equation in the thin shell theory, we can reason out the elastic equations (1.4). 


$$
\begin{aligned}
& \varepsilon_{1}=\frac{1}{A} \frac{\partial u}{\partial \alpha}+\frac{1}{A B} \frac{\partial A}{\partial \beta} v+k_{1} w \\
& \left.\varepsilon_{2}=\frac{1}{B} \frac{\partial v}{\partial \beta}+\frac{1}{A B} \frac{\partial B}{\partial \alpha} u+k_{2} w\right\} \\
& \varepsilon_{12}=\frac{A}{B} \frac{\partial}{\partial \beta}\left(\frac{u}{A}\right)+\frac{B}{A} \frac{\partial}{\partial \alpha}\left(\frac{v}{B}\right) \\
& N_{1}=\frac{E h}{1-\mu^{2}}\left(\varepsilon_{1}+\mu \varepsilon_{2}\right) \\
& N_{2}=\frac{E h}{1-\mu^{2}}\left(\varepsilon_{2}+\mu \varepsilon_{1}\right) \\
& N_{12}=N_{21}=\frac{E h}{2(1+\mu)} \varepsilon_{12} \\
& \frac{1}{A} \frac{\partial u}{\partial \alpha}+\frac{1}{A B} \frac{\partial A}{\partial \beta} v+k_{1} w=\frac{\left(N_{1}-\mu N_{2}\right)}{E h} \\
& \frac{1}{B} \frac{\partial v}{\partial \beta}+\frac{1}{A B} \frac{\partial B}{\partial \alpha} u+k_{2} w=\frac{\left(N_{2}-\mu N_{1}\right)}{E h} \\
& \frac{A}{B} \frac{\partial}{\partial \beta}\left(\frac{u}{A}\right)+\frac{B}{A} \frac{\partial}{\partial \alpha}\left(\frac{v}{B}\right)=\frac{2(1+\mu) N_{12}}{E h}
\end{aligned}
$$

The state of nonmomental theory supposed there are no both flexural moment and torsional moment on the any cross section of the thin shell, that is $M_{1}=M_{2}=M_{12}=M_{21}=0$. Equations (1.1) are simplified (1.5).

$$
\left.\begin{array}{l}
\frac{\partial}{\partial \alpha}\left(B N_{1}\right)-\frac{\partial B}{\partial \alpha} N_{2}+\frac{\partial A}{\partial \beta} N_{12}+\frac{\partial}{\partial \beta}\left(A N_{12}\right)+A B p_{1}=0 \\
\frac{\partial}{\partial \beta}\left(A N_{2}\right)-\frac{\partial A}{\partial \beta} N_{1}+\frac{\partial B}{\partial \alpha} N_{12}+\frac{\partial}{\partial \alpha}\left(B N_{12}\right)+A B p_{2}=0 \\
-k_{1} N_{1}-k_{2} N_{2}+p_{3}=0
\end{array}\right\}
$$

\subsection{The Cylindrical Shell}

The $\alpha$-axis point to the generatrix and the $\beta$-axis point to the circumference of cylindrical shell, then, $k_{1}=0$, $k_{2}=1 / R$ and $A=B=1$, the Gauss-Codazzi conditions are fulfiled. It is shown in figure 3. The balanceable equations and elastic equations of cylindrical shell nonmomental theory are:

$$
\left.\begin{array}{c}
\frac{\partial N_{1}}{\partial \alpha}+\frac{\partial N_{12}}{\partial \beta}+p_{1}=0 \\
\frac{\partial N_{2}}{\partial \beta}+\frac{\partial N_{12}}{\partial \alpha}+p_{2}=0 \\
-N_{2}+R p_{3}=0
\end{array}\right\}
$$

\subsection{The Gyral Shell}

The parameter $C_{1}$ is the curvature center of point $\mathrm{M}$ on the gyral shell generatrix. It is shown in figure 4 . The curvatures are $k_{1}=1 / R_{1}\left(\alpha\right.$ direction) and $k_{2}=1 / R_{2} \quad(\beta$ direction $)$ on the middle surface. At the point $\mathrm{M}$, $d s_{1}=R_{1} d \alpha, d s_{2}=R_{2} \sin \alpha d \beta, A=R_{1}, B=R_{2} \sin \alpha$. 
The Gauss-Codazzi conditions $\quad \frac{\partial}{\partial \beta}\left(k_{1} A\right)=k_{2} \frac{\partial A}{\partial \beta} \quad$ and $\quad \frac{\partial}{\partial \alpha}\left(k_{2} B\right)=k_{1} \frac{\partial B}{\partial \alpha} \quad$ are fulfiled: $\frac{d B}{d \alpha}=\frac{1}{k_{1}} \frac{d k_{2} B}{d \alpha}=R_{1} \frac{d \sin \alpha}{d \alpha}=R_{1} \cos \alpha$, then $\frac{d\left(R_{2} \sin \alpha\right)}{d \alpha}=R_{1} \cos \alpha$. The balanceable equations and elastic equations of gyral shell nonmomental theory are:

$$
\left.\begin{array}{c}
\frac{1}{R_{1}} \frac{\partial N_{1}}{\partial \alpha}+\frac{\operatorname{ctg} \alpha}{R_{2}}\left(N_{1}-N_{2}\right)+\frac{1}{R_{2} \sin \alpha} \frac{\partial N_{12}}{\partial \beta}+p_{1}=0 \\
\frac{1}{R_{1}} \frac{\partial N_{12}}{\partial \alpha}+\frac{2 \operatorname{ctg} \alpha}{R_{2}} N_{12}+\frac{1}{R_{2} \sin \alpha} \frac{\partial N_{2}}{\partial \beta}+p_{2}=0 \\
-\frac{N_{1}}{R_{1}}-\frac{N_{2}}{R_{2}}+p_{3}=0 \\
\frac{1}{R_{1}} \frac{\partial u}{\partial \alpha}+\frac{w}{R_{1}}=\frac{\left(N_{1}-\mu N_{2}\right)}{E h} \\
\frac{1}{R_{2} \sin \alpha} \frac{\partial v}{\partial \beta}+\frac{\cos \alpha}{R_{1} \sin \alpha} u+\frac{1}{R_{2}} w=\frac{\left(N_{2}-\mu N_{1}\right)}{E h} \\
\frac{1}{R_{2} \sin \alpha} \frac{\partial u}{\partial \beta}-\frac{\cos \alpha}{\sin ^{2} \alpha} \frac{\partial v}{\partial \alpha}=\frac{2(1+\mu) N_{12}}{E h}
\end{array}\right\}
$$

The ball shell is the special gyral shell, and $A=B=1, k_{1}=k_{2}=1 / R \quad$ in the ball shell.

\section{The axial symmetrical bending equations of thin shell}

\subsection{The Axial Symmetrical Bending Equations Of Cylindrical Shell}

The internal forces, displacements and strains are axial symmetrical in the cylindrical shell. The internal forces reduce to $N_{1}, N_{2}, M_{1}, M_{2}, Q_{1}$, and the displacements reduce to $u, w$. The axial symmetrical bending equations of the cylindrical shell are:

$$
\frac{d^{4} w}{d \alpha^{4}}+\frac{E h}{R^{2} D} w=\frac{p_{3}}{D}
$$

Dimensionless coordinate is brought in, $\xi=\lambda \alpha$, where $\lambda=\left(\frac{E h}{4 R^{2} D}\right)^{\frac{1}{4}}$, then

$$
\frac{d^{4} w}{d \xi^{4}}+4 w=\frac{4 R^{2}}{E h} p_{3}
$$

The approximate solution of equations (2.2) is made up of the nonmomental theory solution $\left(w^{*}\right)$ and the edge effect solution $\left(w^{0}\right)$, that is,

$$
w=w^{*}+w^{0}=w^{*}+e^{-\xi}\left(C_{1} \cos \xi+C_{2} \sin \xi\right)+e^{\xi}\left(C_{3} \cos \xi+C_{4} \sin \xi\right)
$$

In the equation (2.3), the edge effect solution $\left(w^{0}\right)$ is the solution on the effect of the flexural moment $\left(M_{0}\right)$ and the lateral shearing force $\left(Q_{0}\right)$ that are equally distributed along the boundary at the side of $\alpha=\xi=0$,

$$
\left.\begin{array}{l}
w^{0}=-\frac{M_{0}}{2 \lambda^{2} D} f_{3}(\xi)-\frac{Q_{0}}{2 \lambda^{3} D} f_{4}(\xi) \\
\frac{d w^{0}}{d \alpha}=\lambda \frac{d w^{0}}{d \xi}=\frac{Q_{0}}{2 \lambda^{2} D} f_{1}(\xi)+\frac{M_{0}}{\lambda D} f_{4}(\xi) \\
M_{1}=M_{0} f_{1}(\xi)+\frac{Q_{0}}{\lambda} f_{2}(\xi) \\
Q_{1}=Q_{0} f_{3}(\xi)-2 \lambda M_{0} f_{2}(\xi) \\
M_{2}=\mu M_{1}, N_{2}=\frac{E h}{R} w^{0}
\end{array}\right\}
$$

Where, $f_{1}(\xi)=e^{-\xi}(\cos \xi+\sin \xi), f_{2}(\xi)=e^{-\xi} \sin \xi, f_{3}(\xi)=e^{-\xi}(\cos \xi-\sin \xi), f_{4}(\xi)=e^{-\xi} \cos \xi$.

\subsection{The Axial Symmetrical Bending Equations Of Gyral Shell}

The parameters of the gyral shell, $k_{1}=1 / R_{1}, k_{2}=1 / R_{2}, A=R_{1}, B=R_{2} \sin \alpha$, and on the condition of axial symmetrical bend, $N_{12}=M_{12}=Q_{2}=0$ and $p_{2}=0$, the axial symmetrical bending balanceable equations of the gyral shell are: 


$$
\left.\begin{array}{l}
\frac{1}{R_{1}} \frac{d N_{1}}{d \alpha}-\frac{N_{2}}{R_{1}}+\frac{\operatorname{tg} \alpha}{R_{1}} Q_{1}+\operatorname{tg} \alpha p_{1}=0 \\
\frac{1}{R_{1}} \frac{d M_{1}}{d \alpha}+\frac{M_{2}}{R_{1}}-\operatorname{tg} \alpha Q_{1}=0 \\
-\frac{N_{1}}{R_{1}}-\frac{N_{2}}{R_{2} \sin \alpha}-\frac{\operatorname{ctg} \alpha}{R_{1}} \frac{d Q_{1}}{d \alpha}+p_{3}=0
\end{array}\right\}
$$

The approximate solution of equations (2.5) is made up of the general solution of the homogeneous equation and the special solution of the unhomogeneous equation. The special solution can be solved from the nonmomental theory equations, and the general solution, the edge effect solution, can be solved by hybrid method. Then the equations (2.5) simplified to the equations (2.6).

$$
\left.\begin{array}{l}
\frac{1}{R_{1}} \frac{d N_{1}^{0}}{d \alpha}-\frac{N_{2}^{0}}{R_{1}}+\frac{\operatorname{tg} \alpha}{R_{1}} Q_{1}=0 \\
\frac{1}{R_{1}} \frac{d M_{1}}{d \alpha}+\frac{M_{2}}{R_{1}}-\operatorname{tg} \alpha Q_{1}=0 \\
\frac{N_{1}^{0}}{R_{1}}+\frac{N_{2}^{0}}{R_{2} \sin \alpha}+\frac{\operatorname{ctg} \alpha}{R_{1}} \frac{d Q_{1}}{d \alpha}=0
\end{array}\right\}
$$

The basic functions are supposed, $\omega=\frac{1}{R_{1}}\left(\frac{d w^{0}}{d \alpha}+u^{0}\right), \phi=-R_{2} Q_{1}$.

The differential operator is supposed, $L[\omega]=\left[\frac{R_{2}}{R_{1}} \frac{d}{d \alpha}\left(\frac{1}{R_{1}} \frac{d}{d \alpha}\right)+\frac{\operatorname{ctg} \alpha}{R_{1}} \frac{d}{d \alpha}-\frac{\operatorname{ctg}^{2} \alpha}{R_{2}}\right] \omega$.

The basic differential equations that the axial symmetrical bending edge effect of gyral shell are:

$$
\left.\begin{array}{l}
L(\omega)-\frac{\mu}{R_{1}} \omega=\frac{\phi}{D} \\
L(\phi)+\frac{\mu}{R_{1}} \phi=-E h \omega
\end{array}\right\}
$$

To ball shell, the curvature radius $R_{1}=R_{2}=R$ are constants, and $\phi=-R Q_{1}$, then,

$$
\left.\begin{array}{l}
\frac{d^{2} \omega}{d \alpha^{2}}+\frac{d \omega}{d \alpha} \operatorname{ctg} \alpha-\omega\left(\operatorname{ctg}^{2} \alpha+\mu\right)=\frac{R^{2}}{D} Q_{1} \\
\frac{d^{2} Q_{1}}{d \alpha^{2}}+\frac{d Q_{1}}{d \alpha} \operatorname{ctg} \alpha-Q_{1}\left(\operatorname{ctg}^{2} \alpha-\mu\right)=-E h \omega
\end{array}\right\}
$$

The effect of edge effect reduce rapidly with the distance increase to boundary, then the equations (2.8) simplified to the equations (2.9):

$$
\left.\begin{array}{l}
\frac{d^{2} \omega}{d \alpha^{2}}=\frac{R^{2}}{D} Q_{1} \\
\frac{d^{2} Q_{1}}{d \alpha^{2}}=-E h \omega
\end{array}\right\}
$$

The basic differential equations that the axial symmetrical bending of ball shell are:

$$
\frac{d^{4} Q_{1}}{d \alpha^{4}}+\frac{E h R^{2}}{D} Q_{1}=0
$$

Dimensionless coordinate is brought in, $\eta=\vartheta \alpha$, where $\vartheta=\left(\frac{E h R^{2}}{4 D}\right)^{\frac{1}{4}}$, then,

$$
\frac{d^{4} Q_{1}}{d \alpha^{4}}+4 \vartheta^{4} Q_{1}=0
$$

The internal forces expressions are: 


$$
\left.\begin{array}{l}
N_{1}^{0}=\left[P f_{3}(\eta)+2 M \frac{\vartheta}{R} f_{2}(\eta)\right] \operatorname{ctg} \alpha \\
N_{2}^{0}=2 P \lambda f_{4}(\eta)-2 M \frac{\vartheta^{2}}{R} f_{3}(\eta) \\
M_{1}=-P \frac{\vartheta}{R} f_{2}(\eta)+M f_{1}(\eta) \\
M_{2}=\mu M_{1} \\
Q_{1}=-P f_{3}(\eta)-2 M \frac{\vartheta}{R} f_{2}(\eta)
\end{array}\right\}
$$

Where, $f_{1}(\eta)=e^{-\eta}(\cos \eta+\sin \eta), f_{2}(\eta)=e^{-\eta} \sin \eta, f_{3}(\eta)=e^{-\eta}(\cos \eta-\sin \eta), f_{4}(\eta)=e^{-\eta} \cos \eta$.

\section{The analysisi of torpedo}

The shell of torpedo is made up of ball shell, cylindrical shell, taper shells and other rotary shells by thread coupling, bolt coupling, wedge coupling and hoop coupling. All of them are rigid coupling. The radius of ball shell $R=0.25$, the length of cylindrical shell $L=5.50$, the thickness of shell $h=0.005$, the elastic modulus $E=7.47 \times 10^{10} \mathrm{pa}$, the Poisson's ratio $\mu=0.36$, inner pressure $p_{3}=10^{6} \mathrm{pa}$.

It is shown the force analysis of the coupling of the ball shell and the cylindrical shell in figure 5.

From the balanceable equations of ball shell nonmomental theory, and $R_{1}=R_{2}=R, N_{1}=N_{2}$, obtained the result: $\left(N_{1}^{*}\right)_{\mathrm{B}}=\left(N_{2}^{*}\right)_{\mathrm{B}}=\frac{R p_{3}}{2}$

From the balanceable equations of cylindrical shell nonmomental theory, obtained the result: $\left(N_{2}^{*}\right)_{\mathrm{C}}=R p_{3}, \quad\left(N_{1}^{*}\right)_{\mathrm{C}}=\frac{R p_{3}}{2}$. Obviously, the circumferential direction internal force is not continuous on the coupling circumference, that is $\left(N_{2}^{*}\right)_{\mathrm{B}} \neq\left(N_{2}^{*}\right)_{\mathrm{C}}$, so, there is a direct displacement, and the radial alterations are: $\delta a_{B}=\frac{R^{2} p_{3}}{2 E h}(1-\mu), \delta a_{C}=\frac{R^{2} p_{3}}{E h}\left(1-\frac{\mu}{2}\right)$.

The direct displacement is not continuous, and the difference is $\delta a=\frac{R^{2} p_{3}}{2 E h}$. Thus, there must be $Q_{0}$ and $M_{0}$ that are equally distributed along the circumference, so that the continuousness of the internal force and displacement are ensured. Based on the theory of Timashenko, the rotations of the ball shell and the cylindrical shell are same along the circumference, so $M_{0}=0$, and the discontinuousness is avoided enough by $Q_{0}$.

The direct displacement of the ball shell brought by $Q_{0}$ is $\delta a_{1}^{\prime}=-\frac{Q_{0}}{2 \lambda^{3} D}$, and the cylindrical shell is $\delta a_{2}^{\prime}=\frac{Q_{0}}{2 \lambda^{3} D}$.

The difference is $\delta a^{\prime}=-\frac{Q_{0}}{\lambda^{3} D}$, where $\lambda=\left(\frac{E h}{4 R^{2} D}\right)^{\frac{1}{4}}, \quad D=\frac{E h^{3}}{12\left(1-\mu^{2}\right)}$.

According to the displacement continuous condition, $\delta a+\delta a^{\prime}=0$, then,

$$
Q_{0}=\frac{R^{2} p_{3}}{2 E h} \square \lambda^{3} D=\frac{p_{3} \lambda^{3}}{2} \square \frac{R^{2} D}{E h}=\frac{p_{3} \lambda^{3}}{2} \square \frac{1}{4 \lambda^{4}}=\frac{p_{3}}{8 \lambda} .
$$

The parameters are counted, then,

$$
\begin{gathered}
D=\frac{E h^{3}}{12\left(1-\mu^{2}\right)}=\frac{7.47 \times 10^{10} \times 0.005^{3}}{12\left(1-0.36^{2}\right)}=894, \\
\lambda=\left(\frac{E h}{4 R^{2} D}\right)^{\frac{1}{4}}=\left(\frac{7.47 \times 10^{10} \times 0.005}{4 \times 0.25^{2} \times 894}\right)^{\frac{1}{4}}=36, \quad \vartheta=\left(\frac{E h R^{2}}{4 D}\right)^{\frac{1}{4}}=9
\end{gathered}
$$

When $\xi=0$, then $f_{1}(\xi)=f_{3}(\xi)=f_{4}(\xi)=1, f_{2}(\xi)=0$,

When $\eta=0$, then $f_{1}(\eta)=f_{3}(\eta)=f_{4}(\eta)=1, f_{2}(\eta)=0$,

The results of the cylindrical shell are: 


$$
\left.\begin{array}{l}
N_{1}=\frac{p_{3} R}{2}=1.25 \times 10^{5}, N_{2}=\mu \frac{p_{3} R}{2}+\frac{E h}{R} w=1.875 \times 10^{5} \\
M_{1}=\frac{p_{3}}{8 \lambda^{2}} f_{2}(\xi)=0, M_{2}=\mu M_{1}=0 \\
Q_{1}=\frac{p_{3}}{8 \lambda} f_{3}(\xi)=3472
\end{array}\right\}
$$

The results of the ball shell are:

$$
\begin{aligned}
& N_{1}=\frac{p_{3} R}{2}=1.25 \times 10^{5}, N_{2}=\frac{p_{3} R}{2}+\frac{p_{3} \vartheta}{4 \lambda} f_{3}(\eta)=1.875 \times 10^{5} \\
& M_{1}=-\frac{p_{3} R}{8 \lambda \vartheta} f_{2}(\eta)=0, M_{2}=\mu M_{1}=0 \\
& Q_{1}=-\frac{p_{3}}{8 \lambda} f_{3}(\eta)=-3472
\end{aligned}
$$

As a result, the circumferential direction internal force is not continuous on the coupling circumference.

\section{References}

P. Smithmaitrie \& H.S. Tzou. (2004). Micro-control actions of actuator patches laminated on hemispherical shells. Journal of Sound and Vibration. 277, 157-164.

J.H. Ding \& H.S. Tzou. (2004). Micro-electromechanics of sensor patches on free paraboloidal shell structronic systems Mechanical Systems and Signal Processing. 18. 367-380.

Xu, zhilun. (1982). Elastomechanics. The High Education Press.

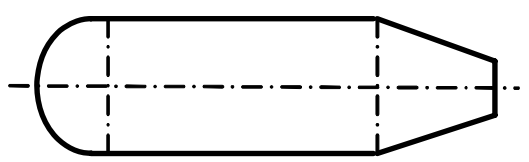

Figure 1. AUV shell

\section{Figure 4. Gyral}

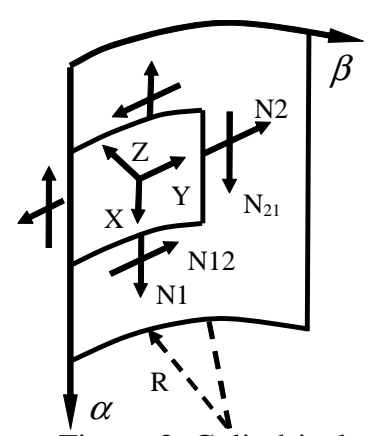

Figure 3. Cvlindrical
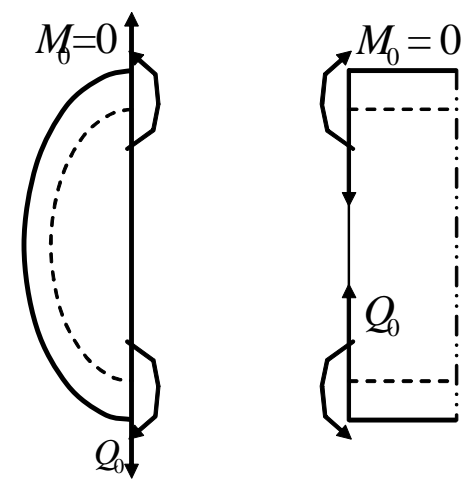

Figure 5. Force analysis of coupling 